\title{
Multi-band Direct-Detection Transmission over an Ultrawide Bandwidth Hollow-Core NANF
}

\author{
Yang Hong, Member, IEEE, Hesham Sakr, Natsupa Taengnoi, Kyle R. H. Bottrill, Thomas D. Bradley, \\ John R. Hayes, Gregory T. Jasion, Hyuntai Kim, Naresh K. Thipparapu, Yu Wang, Andrey A. \\ Umnikov, Jayanta K. Sahu, Francesco Poletti, Periklis Petropoulos, Fellow, OSA, and \\ David J. Richardson, Fellow, IEEE
}

\begin{abstract}
In this paper, we report high-speed multi-band direct-detection (DD) transmission over a hollow-core nested antiresonant nodeless fiber (NANF). Thanks to the ultrawide bandwidth of the NANF, we demonstrate dual-band transmission across the $\mathrm{O}$ - and $\mathrm{C}$-bands over a $\sim 1-\mathrm{km}$ length of a hollow-core fiber for the first time. Eight wavelength-division multiplexed (WDM) channels were transmitted using $100-\mathrm{Gb} / \mathrm{s} / \lambda$ Nyquist 4-ary pulse-amplitude modulation (PAM4) signals, which to the best of our knowledge, is the highest aggregate capacity ever transmitted in a DD hollow-core fiber-based transmission system. Optical pre-amplification was adopted for signal reception in both bands, achieved using an in-house built bismuth-doped optical fibre amplifier (BDFA) and a commercial erbium-doped fiber amplifier (EDFA) in the $\mathrm{O}$ - and $\mathrm{C}$-band, respectively. We further demonstrate beyond $100-\mathrm{Gb} / \mathrm{s} / \lambda$ adaptively-loaded discrete multitone (DMT) transmission over the $\mathrm{S}+\mathrm{C}+\mathrm{L}-$ bands using the same NANF, without the use of optical amplification. Our experiments show that apart from fiber loss, the use of the NANF did not introduce any additional transmission penalties. The demonstrated results validate the ultrawide bandwidth and excellent modal purity of the fabricated NANF, which allow beyond $100-\mathrm{Gb} / \mathrm{s} / \lambda$ penalty-free transmission over multiple bands, highlighting the potential of this fiber technology for high-speed short- to intermediate-reach applications.
\end{abstract}

Index Terms-Microstructured Fibers; Hollow-core Fibers; Ultra-wideband Transmission; WDM Systems; Direct-detection.

\section{INTRODUCTION}

$\mathrm{D}$ ATA traffic in optical networks has experienced rapid growth in recent years, supporting capacity demands for data-hungry services, such as ultra-high resolution video applications and virtual reality, in both wireline and wireless

Manuscript was received XX xx, 2019. This work was supported by the UK's EPSRC under the Airguide Photonics Programme grant (EP/P030181/1), the COALESCE project (EP/P003990/1), as well as through the ERC Lightpipe project (682724). (Corresponding author: Yang Hong).

The authors are with the Optoelectronics Research Centre, University of Southampton, Southampton SO17 1BJ, UK. (e-mails: y.hong@soton.ac.uk; h.sakr@soton.ac.uk; ntla15@soton.ac.uk; k.bottrill@soton.ac.uk; t.bradley@soton.ac.uk; jrh@orc.soton.ac.uk; g.jasion@soton.ac.uk; h.kim@soton.ac.uk; nkt1d17@soton.ac.uk; yw9n17@soton.ac.uk; a.a.umnikov@soton.ac.uk; jks@orc.soton.ac.uk; frap@orc.soton.ac.uk; pp@orc.soton.ac.uk; djr@orc.soton.ac.uk)

Color versions of one or more of the figures in this paper are available online at http://ieeexplore.ieee.org.

Digital Object Identifier: XXXXXXX communication systems [1]. Notably, intra-datacenter traffic with a typical link distance shorter than $2 \mathrm{~km}$ accounts for more than $80 \%$ of global traffic [2-3]. Such links require ultrahigh-speed interconnections $(>100 \mathrm{~Gb} / \mathrm{s} / \lambda)$ and significant experimental progress has already been achieved in this direction [4-5]. Even though coherent transmission systems exhibit superior performance [6], they generally result in a complex, power-hungry and costly system architectures, which have so far been deemed unsuitable for such short-distance implementations. Therefore, research on both digital signal processing (DSP) technologies [7-9] and the associated optical and electronic hardware [10-12] has aimed at improving the performance of the much simpler intensity modulation direct-detection (IM/DD) solutions. In [7-9], advanced modulation formats including 4-ary pulse amplitude modulation (PAM4) and discrete multi-tone (DMT), and their associated DSP algorithms have been demonstrated. PAM4 signaling, which offers a good compromise between scalability in data rate, complexity and cost of implementation, has been favored as the modulation format for intra-datacenter applications, and $4 \times 100$ G PAM 4 transceivers are expected to be commercialized in the near future [2]. A representative recent example of the remarkable progress that has been achieved in short-reach optical systems using PAM4 was reported in [7], where PAM4 signals were multiplexed on orthogonal polarizations and an additional inter-polarization phase modulation was combined with them to enhance the aggregate capacity. On the other hand, albeit at the expense of some increased implementation complexity, the DMT scheme provides the capability to either maximize the achievable capacity or minimize the bit-error-rate (BER) of the system by employing adaptive bit-and-power loading algorithms. Beyond $100-\mathrm{Gb} / \mathrm{s} / \lambda$ DMT transmission was demonstrated in the C-band over 1.6-km of standard single-mode fibre (SSMF) [8]. In [9], it was shown that the transmission performance of adaptively-loaded DMT is superior to that of the PAM formats under the same system configuration.

In addition, the use of novel fiber technologies has also been considered in order to boost the capacity of short-reach systems. For example, multicore fibers (MCF) have been utilized effectively to achieve multiple single-mode paths simultaneously within a single fiber, which enables a substantial improvement in achievable capacity [13]. A 
125 - $\mu \mathrm{m}$-cladding eight-core fibre was reported in [14], through which a $25-\mathrm{Gb} / \mathrm{s} / \lambda$ WDM transmission in the O-band was demonstrated. Further improvement in the aggregate capacity of the MCF-based transmission was reported in [15], where wavelengths both in the O-band and in the $\mathrm{C}$ - and L-bands were simultaneously adopted. A radically different route offering promising benefits in short-reach interconnects is the use of the hollow-core fibers (HCFs). The operation of HCFs relies on fundamentally different propagation principles than conventional solid-core optical fibers, since the light is guided in air (i,e, within a low refractive index region [16-18]). This characteristic of HCFs introduces a variety of unique advantages over conventional fibers, including low latency, ultralow nonlinearity and the potential for ultralow loss, which make HCF an attractive medium for high-speed telecom applications.

To date, most high-speed HCF-based data transmission experiments were demonstrated using coherent detection [19-23], which is however mainly used in long-haul applications. In [19], up to 73.7-Tb/s transmission over a 310-m length of photonic bandgap HCF was reported. A recent demonstration showed multi-terabit/s nonlinearity-free coherent transmission over a 270-m-long antiresonant HCF and numerically validated the advantages of HCF in long-haul applications [20]. Although relatively long-distance (up to 340 $\mathrm{km}$ ) HCF-based transmission experiments have been conducted using a re-circulating loop in $[22,23]$, current challenges in both fibre loss and the fabrication of uniform fibre structure over long lengths currently constrain realistic HCF transmission trials to relatively short distances. Nonetheless, even at their current stage of development, HCFs have the potential to offer significant impact in various short-reach applications, especially in the area of intra-datacenter interconnects, where ultra-low latency is crucial. In [24], we recently demonstrated a novel nested antiresonant nodeless fibre (NANF), which was capable of supporting effective single-mode transmission across the O-, E-, S-, C- and L-band.

Using $1 \mathrm{~km}$ of this NANF, and taking advantage of recent developments in bismuth-doped fiber amplification technology for the O-band, we present here our experiments on dual $\mathrm{O}+\mathrm{C}-$ band $100-\mathrm{Gb} / \mathrm{s} / \lambda$ wavelength division multiplexing (WDM) transmission. To provide a more comprehensive analysis of the transmission performance of the NANF as a short-reach transmission fibre, we also present results obtained using adaptively-loaded DMT transmission over the same fibre length, which capitalize on the excellent modal purity of the fabricated NANF to demonstrate penalty-free beyond $100-\mathrm{Gb} / \mathrm{s}$ transmission. This paper encompasses the preliminary results presented in $[25,26]$, and provides detailed background and technical information, and additional transmission results, as well as new insight and discussion. Specifically, the additional experimental results include: (1) the comparison of electrical spectra of the dual $\mathrm{O}+\mathrm{C}$-band transmission system with and without pre-emphasis; (2) the signal-to-noise ratio (SNR) comparisons of the WDM channels in both the O-band and the C-band; (3) the average SNR and maximum achievable spectral efficiency (SE) of the hollow-core NANF based system over
$\mathrm{S}+\mathrm{C}+\mathrm{L}-$ bands; and (4) the comparison of the average SNR for the system after back-to-back (B2B) and NANF transmission. The rest of this paper is structured as follows. In Section II, the properties of the fabricated ultrawide bandwidth NANF are briefly described. In Section III, we present the dual O+C-band $100-\mathrm{Gb} / \mathrm{s} / \lambda$ PAM4 WDM transmission experiments. We present the DSP that we used in detail and provide additional analysis of the SNR performance in both bands. In Section IV, experimental results on adaptively-loaded DMT transmission in the $\mathrm{S}+\mathrm{C}+\mathrm{L}-$ bands over the NANF are presented and discussed. Finally, Section V concludes this work.

\section{THE UlTRAWIDE BANDWIDTH NANF}

In this section, we briefly describe the properties of the fully spliced NANF used in this work. Fig. 1 shows an illustration of the SMF-connectorized $\sim 1-\mathrm{km}$ NANF and the corresponding cross-section scanning electron microscope (SEM) images of the fiber structure. To form a $\sim 1-\mathrm{km}$ length of fiber, two structurally matched spools of NANF with respective lengths $340 \mathrm{~m}$ (NANF\#1) and $620 \mathrm{~m}$ (NANF\#2) were spliced together with a splice loss of around $0.1 \mathrm{~dB}$. To incorporate the NANF in an SMF-based optical system, the two ends of the NANF were spliced to SMF patch cords via an arrangement of mode field adaptors (MFAs) that expanded the mode field of the SMF, and intermediate mode area fibers (IMAFs) that had a similar mode field area to the NANF, to reduce the splicing loss between SMF and NANF [24, 27].

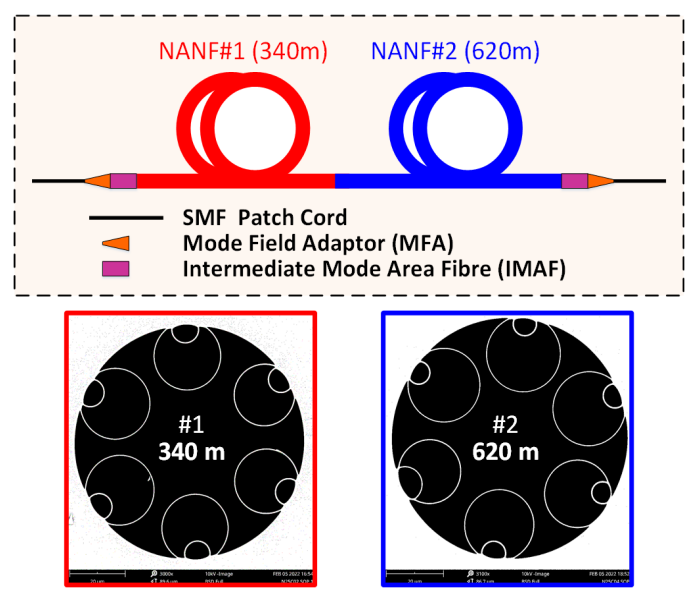

Fig. 1. Illustration of the SMF-connectorized $\sim 1-\mathrm{km}$ NANF assembly. Insets: SEM images of the fabricated NANFs used in this work.

The NANF had a core with a diameter of $35 \pm 0.5 \mu \mathrm{m}$, which was surrounded by six nested tubes, as depicted in Fig. 1. The outer diameter of the outer tubes was $23.7 \pm 1.5 \mu \mathrm{m}$ and the thickness of the struts was $433.5 \pm 28.5 \mathrm{~nm}$. For the inner tubes, the outer diameter and the thickness of their struts were $9.45 \pm 0.55 \mu \mathrm{m}$ and $485.5 \pm 28.5 \mathrm{~nm}$, respectively. The azimuthal gaps between the outer tubes ranged from $6.1 \mu \mathrm{m}$ to $7.7 \mu \mathrm{m}$, and this was consistent throughout the whole fiber length during the stack-and-draw process $[24,28]$. The NANF was measured to exhibit an ultrawide $3-\mathrm{dB}$ bandwidth of $700 \mathrm{~nm}$ (ranging from $1240 \mathrm{~nm}$ to $1940 \mathrm{~nm}$ ), which substantially exceeds the wavelength range of the $\mathrm{O}+\mathrm{E}+\mathrm{S}+\mathrm{C}+\mathrm{L}-$ bands. A 


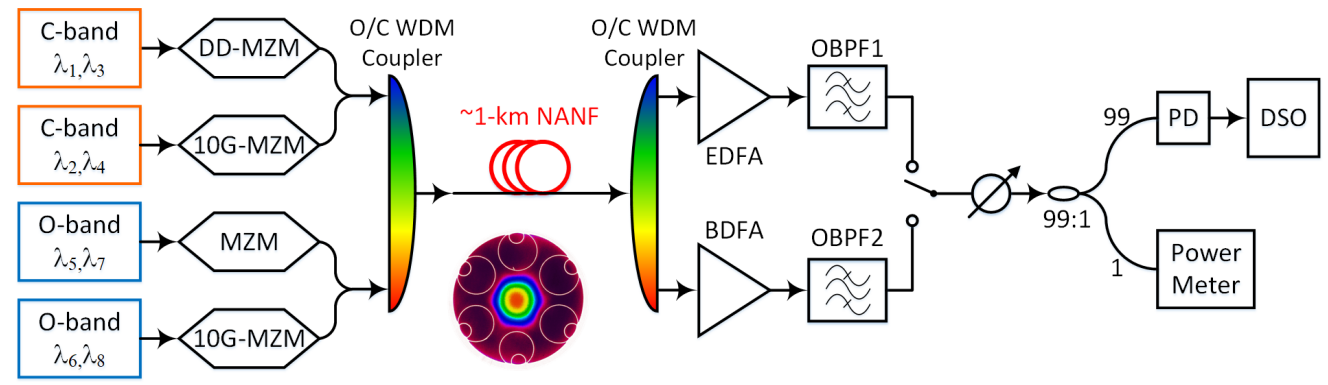

Fig. 2. Experimental setup of the dual O+C-band transmission system over $\sim 1-\mathrm{km}$ length of NANF. Inset: the cross-sectional image of the NANF.

minimum loss of $6.6 \mathrm{~dB} / \mathrm{km}$ was achieved at $1550 \mathrm{~nm}$, and a loss of less than $10 \mathrm{~dB} / \mathrm{km}$ was measured for wavelengths between 1297-1860 nm [24], which is the lowest loss ever reported for HCFs with a bandwidth $>700 \mathrm{~nm}$. Compared to the ideal designed fiber structure, some structural non-uniformities, such as non-ideal inter-tube gaps or slightly misaligned inner capillaries, existed in our fabricated NANF. These non-uniformities degraded the loss and limited the bandwidth of the fiber. Furthermore, our numerical results showed that by refining the fiber structure, e.g. reducing the size of the inter-tube gap to $\sim 2 \mu \mathrm{m}$, a sub- $\mathrm{dB} / \mathrm{km}$ loss can be achieved, which will require further refinement of our fabrication technology. An in-depth analysis of the loss contributions of the fabricated NANF and routes to further reducing its loss were reported in [28]. In terms of high order mode content/mode coupling within the fabricated NANF, it was demonstrated that the NANF is effectively single-mode. The $\mathrm{LP}_{11}$ mode was suppressed to the noise level $(\sim-75 \mathrm{~dB})$ after transmission over $350 \mathrm{~m}$ of the NANF (NANF\#1 in Fig. 1), as characterized in [24].

Taking into account all of the additional losses from splicing and mode field mismatch in the 1-km SMF-connectorized NANF, the total insertion loss ranged from 10 to $12 \mathrm{~dB}$ from the $\mathrm{O}$ to the $\mathrm{L}$-band. Furthermore, a low polarization dependent loss (PDL) of $\sim 0.5 \mathrm{~dB}$ (maximum PDL of $0.6 \mathrm{~dB}$ ) was observed [26], indicating that the SMF-connectorized NANF could be used in transmission experiments without any need for control of the polarization at the fiber's input. It is also worth noting that prior evaluation of bending in hollow-core NANF indicated that the fiber can be coiled, e.g. in splice trays, without incurring an excessive additional loss at $1550 \mathrm{~nm}$ [27]. Therefore, it is anticipated that bending should not be an issue when using the hollow-core NANF in practical systems.

\section{DUAL O+C-BAND WDM TRANSMISSION OVER THE NANF}

In this section, we present multi-band WDM transmission experiments over the NANF, which were conducted to both validate and utilize its ultrawide bandwidth. We used the two most commonly used bands in optical communication applications, i.e., the O- and C-band, to simultaneously transmit WDM $100-\mathrm{Gb} / \mathrm{s} / \lambda$ Nyquist PAM4 signals. The experiments highlight the spectrally-uniform loss performance of the fabricated $\sim 1-\mathrm{km}$ length of NANF.

\section{A. Setup of the $O+C$-band Transmission over the NANF}

Fig. 2 shows the experimental setup of the NANF-based dual $\mathrm{O}+\mathrm{C}$-band WDM system. The C-band transmitter employed four lasers at $1549.32 \mathrm{~nm}, 1550.12 \mathrm{~nm}, 1550.92 \mathrm{~nm}, 1551.72$ $\mathrm{nm}$ (100-GHz-spaced WDM channels on the ITU grid). The odd and even channels were fed into two separate Mach-Zehnder modulators (MZMs), respectively, to produce un-correlated modulated optical signals. The outputs of the two MZMs were combined via a 3-dB optical coupler. The resulting C-band WDM signal was then fed into an O/C WDM coupler. Similarly, in the O-band, four lasers working at $1330.6 \mathrm{~nm}$, $1341.8 \mathrm{~nm}, 1351.16 \mathrm{~nm}$ and $1359.88 \mathrm{~nm}$ were combined into odd and even channels and sent to two more MZMs for optical modulation. The outputs of these two MZMs were coupled together via another 3-dB coupler and then also fed to the $\mathrm{O} / \mathrm{C}$ WDM coupler. Finally, the coupled WDM signal comprising four channels in both the O- and C-band (eight channels total) were directly launched into the NANF.

After transmission in the NANF, at the receiver, another $\mathrm{O} / \mathrm{C}$ WDM coupler was used to de-multiplex the two bands. The C-band optical signal was first pre-amplified by a commercial erbium-doped fiber amplifier (EDFA). Subsequently, a C-band tunable filter with a bandwidth of $0.8 \mathrm{~nm}$ was adopted to select the target channel for performance evaluation. A similar setup was implemented in the O-band, except that an in-house built bismuth-doped optical fiber amplifier (BDFA) and an O-band tunable filter with a 1.2-nm bandwidth were used for pre-amplification and channel selection, respectively. A detailed characterization of the BDFA can be found in [29]. An optical attenuator was used to control the received optical power (ROP), $99 \%$ of which was fed into a dual-window photodetector (PD, Finisar XPDV2320R). The remaining 1\% was used for power monitoring. A single PD was used in the experiments to detect the $\mathrm{C}$ - or O-band signals. The specified responsivity of the $\mathrm{PD}$ was $0.65 \mathrm{~A} / \mathrm{W}$ and $0.45 \mathrm{~A} / \mathrm{W}$ in the $\mathrm{C}$ and O-band, respectively. The output of the PD was captured by a digital storage oscilloscope (DSO, Agilent DSO-X 93204A) for offline DSP. Note that due to the limited availability of high-bandwidth MZMs, two 10G-class MZMs were used in the setup (one in each band), as depicted in Fig. 2. During the simultaneous $\mathrm{O}+\mathrm{C}$-band WDM transmission, the two 10G-class MZMs were driven by 50-Gb/s PAM4 signals whilst the dual-drive MZM (DD-MZM) used in the C-band and the 


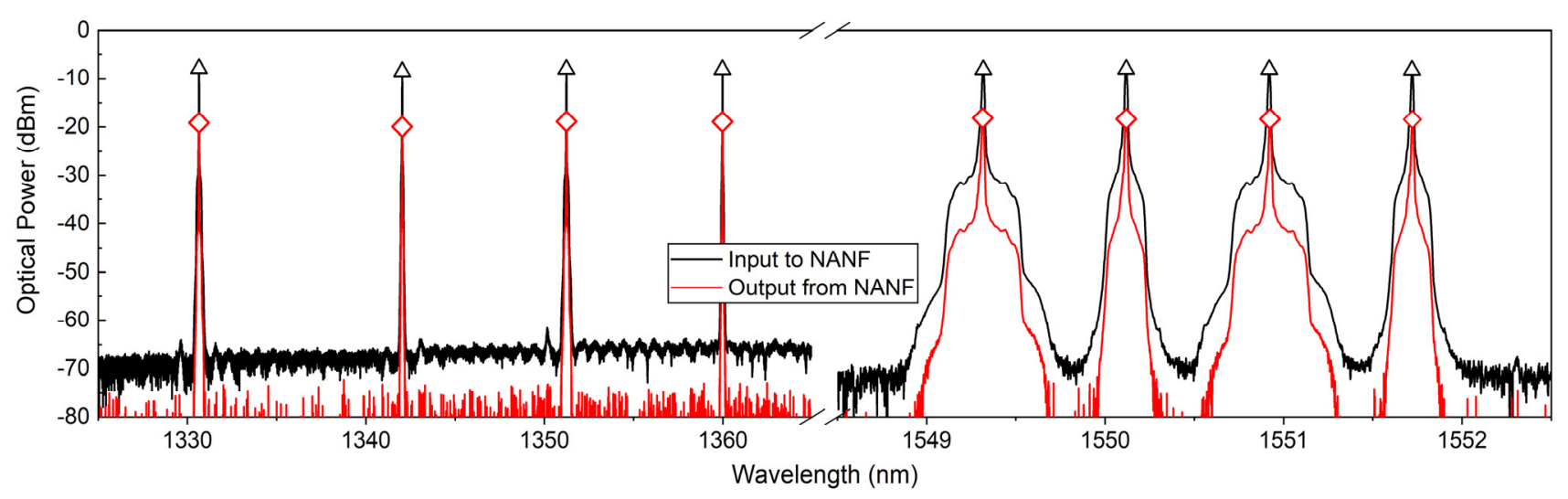

Fig. 4. Optical spectra of the O- and C-band WDM signals at the input and output of the NANF. Note that different $x$-axis scales are used for the two bands for clearer presentation.

single-drive MZM used in the O-band were driven by $100-\mathrm{Gb} / \mathrm{s}$ PAM4 signals. Nevertheless, during the characterization and measurements, the modulators of the odd/even channels were swapped, and only 100-Gb/s PAM4 transmission results were captured for all eight WDM channels.

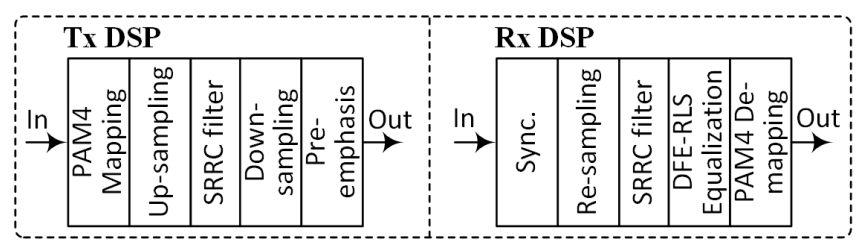

Fig. 3. Diagram of the offline DSP for the dual $\mathrm{O}+\mathrm{C}-$ bands transmission.

Fig. 3 shows the diagram of the offline DSP used in both bands to generate the Nyquist PAM4 signals. At the transmitter, the input binary bits were first mapped into PAM4 symbols, followed by an up-sampling operation. The up-sampled signal was then filtered by a square-root-raised-cosine (SRRC) filter with a roll-off factor of 0.1 . Subsequently, the filtered signal was down-sampled and pre-emphasized. Finally, the generated signals were fed into a four-channel arbitrary waveform generator (AWG, Keysight M8196A) to produce the $50-\mathrm{Gb} / \mathrm{s}$ and $100-\mathrm{Gb} / \mathrm{s}$ PAM4 signals, using a fixed sampling rate of 90 $\mathrm{GSa} / \mathrm{s}$. The pre-emphasis was performed at the transmitter by applying a linear inverse finite impulse response (FIR) filter with a tap number of 127 , and the tap coefficients were optimized under the mean-squared-error criterion. At the receiver, the sampling rate of the DSO was fixed at $80 \mathrm{GSa} / \mathrm{s}$. The recorded data were processed by the order of synchronization, re-sampling and SRRC filtering. After that, the resulting signal was equalized by a $\mathrm{T} / 2$-spaced decision-feedback equalizer (DFE) using the recursive least-squares (RLS) algorithm. The forgetting factor of the RLS algorithm was 0.99 , and the numbers of the feedforward and feedback weights of the DFE were 17 and 7, respectively. Finally, the equalized signal was PAM4 de-mapped for BER calculation via error counting.

\section{B. Results of the dual $O+C$-band transmission}

To verify the suitability of the fabricated NANF for dual
$\mathrm{O}+\mathrm{C}$-band data transmission, we first investigated the loss performance of the NANF in both bands. The optical spectra of the $\mathrm{O}$ - and $\mathrm{C}$-band WDM signals at the input and output of the NANF are shown in Fig. 4. A comparison of the power of the channels reveals that the loss performance over the two bands is spectrally uniform $(\sim 10 \mathrm{~dB})$. Note that as aforementioned, the even channels shown in the results of Fig. 4 were modulated with $50-\mathrm{Gb} / \mathrm{s}$ PAM4 signals, hence they occupied a narrower bandwidth. In all of the transmission results that will be shown below, the performance of even channels was investigated only for $100-\mathrm{Gb} / \mathrm{s}$ PAM4 transmission (by swapping the MZMs used in the setup).

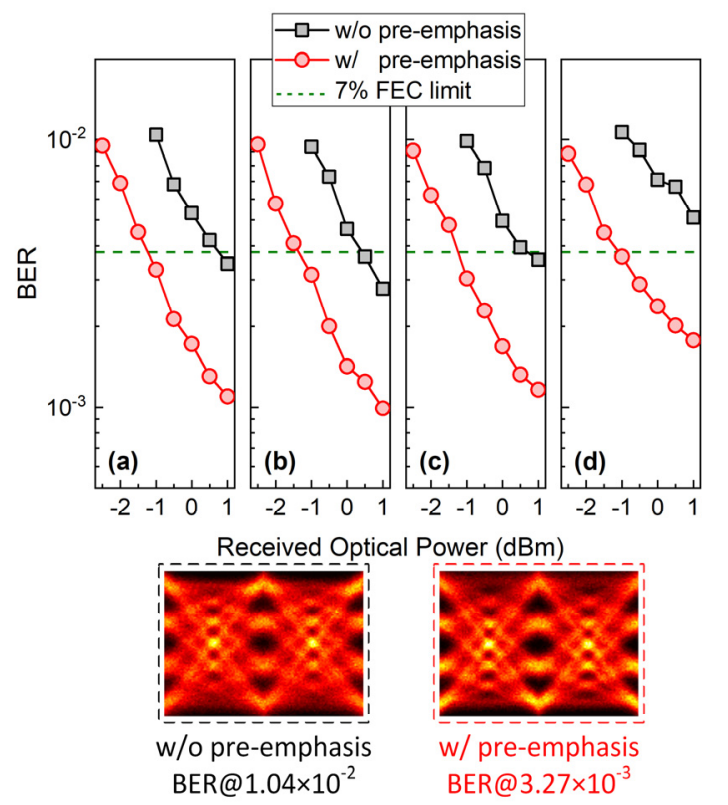

Fig. 5. BER versus ROP in the O-band: (a) $1330.6 \mathrm{~nm}$, (b) $1341.8 \mathrm{~nm}$, (c) $1351.16 \mathrm{~nm}$ and (d) $1359.88 \mathrm{~nm}$. Insets: eye diagrams of recovered PAM4 signals for the 1330.6-nm channel with a ROP of $-1 \mathrm{dBm}$.

Fig. 5 shows the BERs versus received optical powers (ROPs) of the four O-band channels. Thanks to the uniform loss performance of the NANF, similar BERs were achieved for the four channels. When pre-emphasis was not utilized, a ROP greater than $1 \mathrm{dBm}$ was required to achieve a BER lower than 
the $7 \%$ forward error correction (FEC) limit, i.e. $3.8 \times 10^{-3}$. We then implemented pre-emphasis to compensate for the frequency roll-off effect of the overall system, which resulted in significant improvement in the transmission performance.

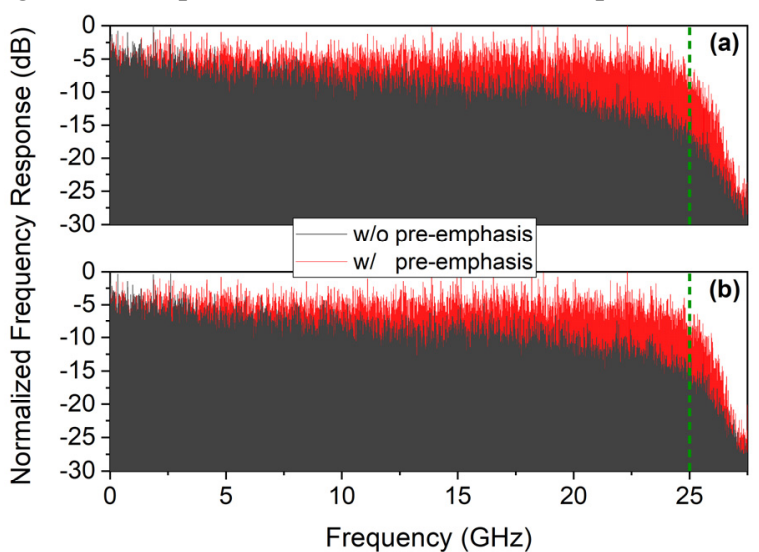

Fig. 6. The electrical spectra of the system with and without pre-emphasis: (a) the 1330.6-nm channel and (b) the 1549.32-nm channel.

The comparison of the electrical spectra of the 1330.6-nm channel (taken as an example) with and without pre-emphasis is shown in Fig. 6(a). It is clear that without using the pre-emphasis, the fading within the signal's bandwidth was more than $10 \mathrm{~dB}$, which resulted from the combined roll-off in frequency response of the transmitter and the receiver. This fading severely degraded the transmission performance of the system. By utilizing the pre-emphasis technique, the frequency roll-off was effectively combated and a flat frequency response could be achieved within the signal's bandwidth (i.e., $25 \mathrm{GHz}$, as labelled in Fig. 6). As a result, the required ROP to attain a $\mathrm{BER}<3.8 \times 10^{-3}$ was relaxed to around $-1 \mathrm{dBm}$ for the O-band channels. For reference, the eye diagrams of recovered PAM4 signals in the $1330.6-\mathrm{nm}$ channel at a ROP of $-1 \mathrm{dBm}$ are also presented in Fig. 5. The corresponding BERs for the cases with and without the pre-emphasis were $1.04 \times 10^{-2}$ and $3.27 \times 10^{-3}$ at this power level, respectively. The SNRs versus ROPs of the O-band channels are given in Fig. 7. It can be seen that for a certain ROP, pre-emphasis could provide more than a 1-dB improvement in the SNR. For example, at a ROP of $-1 \mathrm{dBm}$, the SNR of the 1330.6-nm channel was improved from around $\sim 14.6 \mathrm{~dB}$ to $\sim 15.8 \mathrm{~dB}$. Furthermore, it is seen in Fig. 5 and Fig. 7 that the BER/SNR performance in the 1359.88-nm channel was slightly worse than that of the other channels. This is mainly attributed to the extra loss of the WDM coupler (whose operation range is from $1260 \mathrm{~nm}$ to $1360 \mathrm{~nm}$ ) and the marginally lower gain of the BDFA at that wavelength [29].

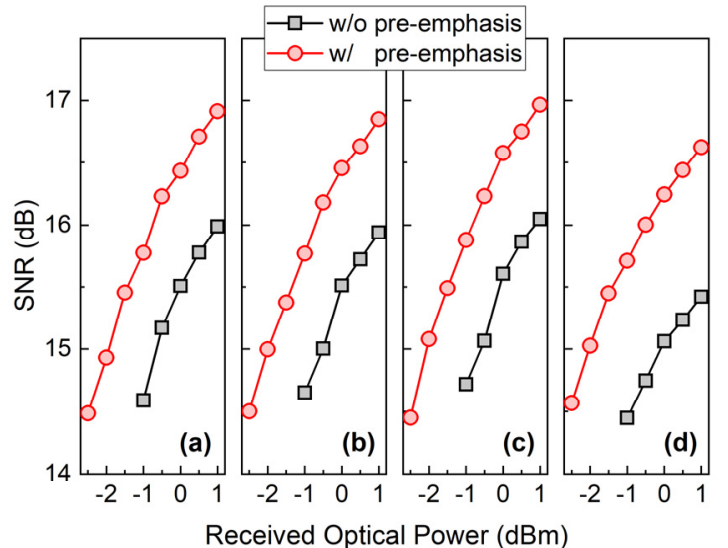

Fig. 7. SNR versus ROP in the O-band: (a) $1330.6 \mathrm{~nm}$, (b) $1341.8 \mathrm{~nm}$, (c) $1351.16 \mathrm{~nm}$ and (d) $1359.88 \mathrm{~nm}$

The BERs versus ROPs of the four channels in the C-band are shown in Fig. 8. It is seen that without using pre-emphasis, all channels exhibited a BER lower than the 7\% FEC limit when the ROP was greater than $-3 \mathrm{dBm}$. All of the four channels gave similar BER performance, owing to the comparable loss performance of the NANF at these wavelengths. Similar to the O-band case, by using pre-emphasis, significant BER reduction could be achieved. For example, taking the 7\% FEC limit as the BER threshold, around 1.5-dB ROP sensitivity improvement could be attained across all channels when using pre-emphasis. For reference, the corresponding eye diagrams are also presented in Fig. 8. The corresponding measurements of SNR versus ROP of the C-band channels are shown in Fig. 9. It is seen that pre-emphasis led to around 1.5-dB SNR improvement for all channels. For instance, at a ROP of $-4.5 \mathrm{dBm}$, the SNR of the 1549.32-nm channel was enhanced from $\sim 14.7 \mathrm{~dB}$ to $\sim 16.2 \mathrm{~dB}$.

By comparing the BER/SNR results of the two bands, we see that the channels in the O-band required a higher ROP than the C-band channels to achieve a similar BER/SNR performance. Since the NANF exhibits comparable loss in both bands (Fig. 4) and its length is $\sim 1-\mathrm{km}$ long, we attribute the increase in the ROP requirement to the performance difference of the transceiver devices used for the two bands, among which the aforementioned lower responsivity of the PD and higher bandwidth of the optical filter adopted in the O-band are the dominant factors.

We note that since the optical beams within the NANF are air-guided, the chromatic dispersion (CD) in the hollow-core NANF takes ultra-low values across the ultrawide bandwidth. For the hollow-core NANF used in our experiments, the simulated CD values ranged from $\sim 1.48$ to $\sim 2.26 \mathrm{ps} / \mathrm{nm} / \mathrm{km}$ for wavelengths ranging from $1260 \mathrm{~nm}$ to $1625 \mathrm{~nm}$. Hence, the CD has a negligible effect on the performance of the dual $\mathrm{O}+\mathrm{C}$-band transmission over the $\sim 1-\mathrm{km}$ length of NANF. Recent experiments have demonstrated that high-speed NANF transmission is tolerant to CD-induced power fading effects, even when extending the reach further to several kilometers [30]. However, since the CD in the fiber is cumulative, the performance of the dual $\mathrm{O}+\mathrm{C}$-band NANF transmission will 
eventually be affected by the CD-induced power fading if a much longer reach is considered. In this case, dispersion-tolerant signal formats, e.g., single-sideband modulation, or coherent detection can be adopted to significantly extend the reach of the dual $\mathrm{O}+\mathrm{C}-$ band NANF transmission.

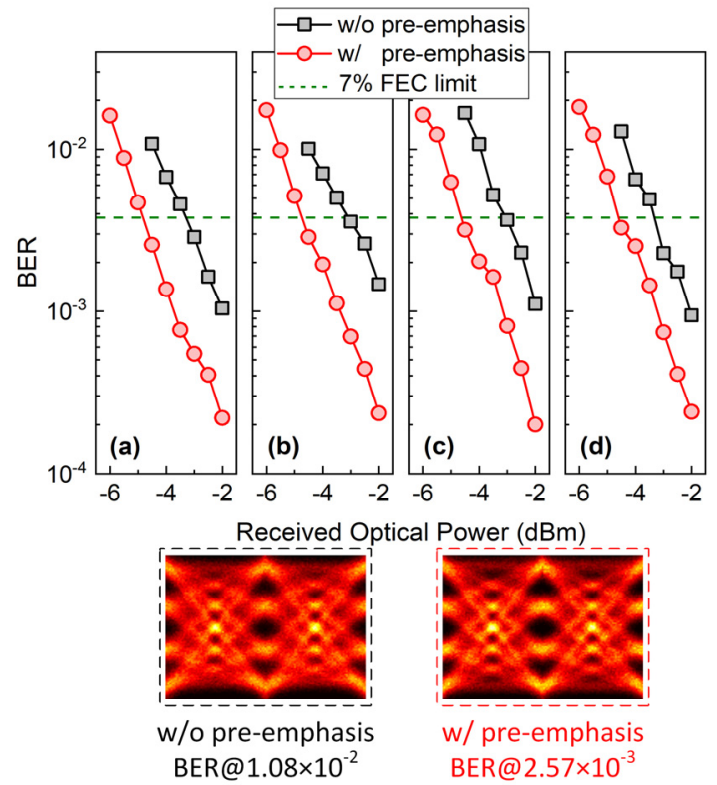

Fig. 8. BER versus ROP in the C-band: (a) $1549.32 \mathrm{~nm}$, (b) $1550.12 \mathrm{~nm}$, (c) $1550.92 \mathrm{~nm}$ and (d) $1551.72 \mathrm{~nm}$. Insets: eye diagrams of recovered PAM4 signals for the $1549.32-\mathrm{nm}$ channel with a ROP of $-4.5 \mathrm{dBm}$.

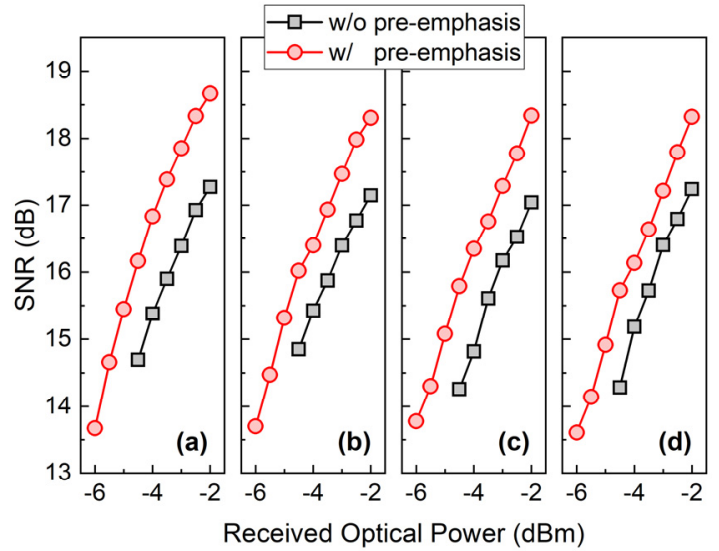

Fig. 9. SNR versus ROP in the C-band: (a) $1549.32 \mathrm{~nm}$, (b) $1550.12 \mathrm{~nm}$, (c) $1550.92 \mathrm{~nm}$ and (d) $1551.72 \mathrm{~nm}$.

\section{ADAPTIVELY-LOADED DMT TRANSMISSION OVER S + C+L-BANDS IN THE NANF}

We next changed the modulation format from Nyquist PAM4 to adaptively-loaded DMT and conducted transmission experiments in the NANF across the S-, C- and L-bands. The adaptively-loaded DMT facilitates a more efficient use of the electrical bandwidth by assigning different power and numbers of bits to different data subcarriers according to their available SNRs. It therefore allows exploration of the maximum capacity that can be transmitted in this fashion over a given channel under a certain ROP. In this section, we capitalized on the excellent modal purity of the fabricated NANF to demonstrate beyond $100-\mathrm{Gb} / \mathrm{s} / \lambda$, penalty-free, adaptively-loaded DMT transmission. A maximum channel capacity of up to $132.8-\mathrm{Gb} / \mathrm{s} / \lambda$ transmission was achieved without the use of any optical amplification.

\section{A. Experimental setup of the Adaptively-loaded DMT Transmission over the NANF}

Fig. 10 shows the experimental setup and the corresponding DSP diagrams of the adaptively-loaded DMT transmission over the NANF. CW light with a wavelength that was varied between $1461 \mathrm{~nm}$ and $1631 \mathrm{~nm}$ (covering the whole of S-, C-, and L-bands) was fed into a single-drive MZM. The output of the MZM was directly launched into the SMF-connectorized NANF without optical amplification. A high-gain PD (Finisar, XPRV2022A) was used to detect the signal at the output of the NANF. Subsequently, the detected signal was captured by an $80-\mathrm{GSa} / \mathrm{s}$ DSO for further offline DSP. Note that to maximize the capacity, the output power of the $\mathrm{S}+\mathrm{C}+\mathrm{L}$-band tunable laser was maximized at each tested wavelength. The bias voltage of the MZM was also optimized for different wavelengths. It is worth noting that the wavelength range investigated in this section was limited by the availability of the tunable laser. Since the NANF has similar propagation characteristics across the O- to L-band [24], comparable transmission performance can be expected in the O- and E-band as well.

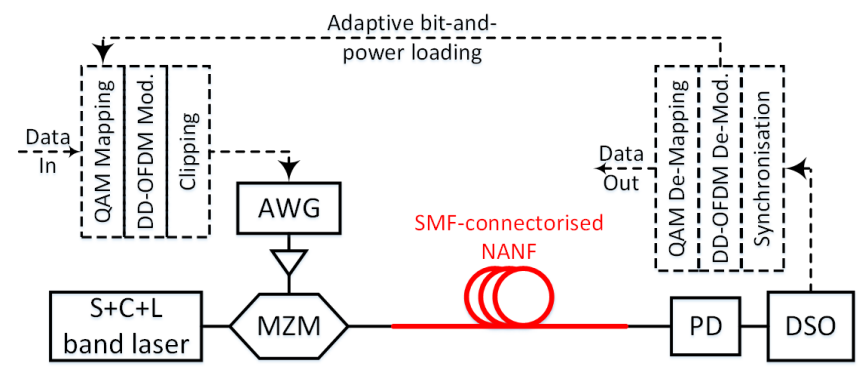

Fig. 10. Experimental setup and the corresponding DSP blocks of the adaptively-loaded DMT transmission over the NANF.

In the offline DSP, at the transmitter, the binary bits were first mapped to quadrature amplitude modulation (QAM) symbols, followed by DD orthogonal frequency division multiplexing (DD-OFDM) modulation [26]. The DD-OFDM modulation included the Hermitian symmetry operation, inverse fast Fourier transform (IFFT) and insertion of the cyclic prefix (CP). The size of IFFT was 512, out of which 240 subcarriers were used for data modulation. The length of CP was $1 / 8$ of one DD-OFDM symbol. The output of the DD-OFDM modulation module was then clipped using a 12-dB clipping ratio before been sent to an $\mathrm{AWG}$. The sampling rate of the AWG was fixed at $70 \mathrm{GSa} / \mathrm{s}$ to generate analogue signals with an electrical bandwidth of $32.8125 \mathrm{GHz}$. The RF signals produced by the AWG were amplified by an RF amplifier, and then used to drive the MZM. In the receiver DSP, the captured signal was first synchronized, followed by DD-OFDM demodulation. The processing of the DD-OFDM demodulation module consisted of low-pass filtering, removal of the CP, FFT, channel estimation, one-tap equalization and extraction of the 
data-carrying subcarriers. After the DD-OFDM demodulation, the resulting signal was QAM de-mapped for BER calculation. Pilot DD-OFDM transmission was first conducted to estimate the SNR conditions of the data subcarriers, based on which the adaptive loading algorithm [26, 31] was implemented to maximize the capacity of the system using the $7 \%$ FEC limit as the BER threshold.

\section{B. Results of the $S+C+L$-bands transmission over the NANF}

Since the maximum output power delivered by our tunable laser varied with wavelength, we first investigated the transmission performance of the system using a $65.625-\mathrm{Gb} / \mathrm{s}$ 4QAM-DD-OFDM signal at the maximum power per wavelength, which also served as the pilot transmission for the estimation of the channel state information. Here, all the data subcarriers were loaded with 4QAM symbols (which corresponded to a data rate of $65.625 \mathrm{~Gb} / \mathrm{s}$ ), and no power loading was used. The results are shown in Fig. 11. It is seen that a higher ROP was obtained at wavelengths around the C-band. This was due to the output power characteristics of the tunable laser rather than the NANF. As expected, a higher ROP resulted in a lower BER, as shown in Fig. 11(a). When a ROP > $-10.5 \mathrm{dBm}$ was available, the corresponding BER was below the $7 \%$ FEC limit. The results of the corresponding average SNR, i.e., average SNR among all data subcarriers, versus wavelength are presented in Fig. 11(b), where a similar trend to that of the ROP is observed. Based on the estimated SNRs, adaptive bit-and-power loading was implemented at each wavelength to maximize the SE and hence the capacity. The maximized SE achieved at each wavelength is also shown in Fig. 11(b). A maximum SE of $\sim 4.046 \mathrm{bits} / \mathrm{s} / \mathrm{Hz}$ was achieved at $1551 \mathrm{~nm}$, which corresponded to a maximum capacity of around $132.8 \mathrm{~Gb} / \mathrm{s}$.
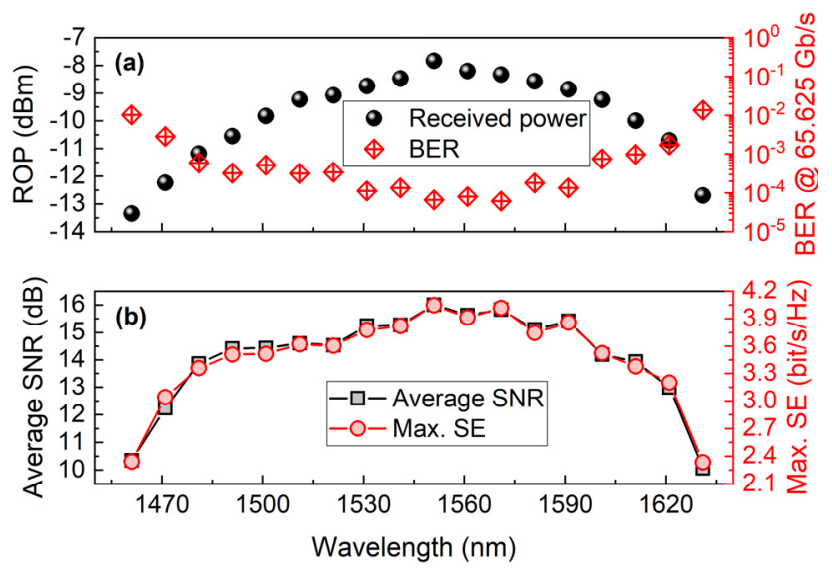

Fig. 11. (a) ROP and BER versus wavelength, and (b) average SNR and maximum $\mathrm{SE}$ versus wavelength.

Fig. 12 shows the corresponding data rates and BERs after transmission for different wavelengths with the maximized SEs using the adaptively-loaded bit-and-power allocation results. It is shown that without optical amplification, beyond $100-\mathrm{Gb} / \mathrm{s}$ transmission could be achieved for wavelengths ranging from $\sim 1470 \mathrm{~nm}$ to $\sim 1620 \mathrm{~nm}$, whilst the corresponding BERs were all below the $7 \%$ FEC limit.

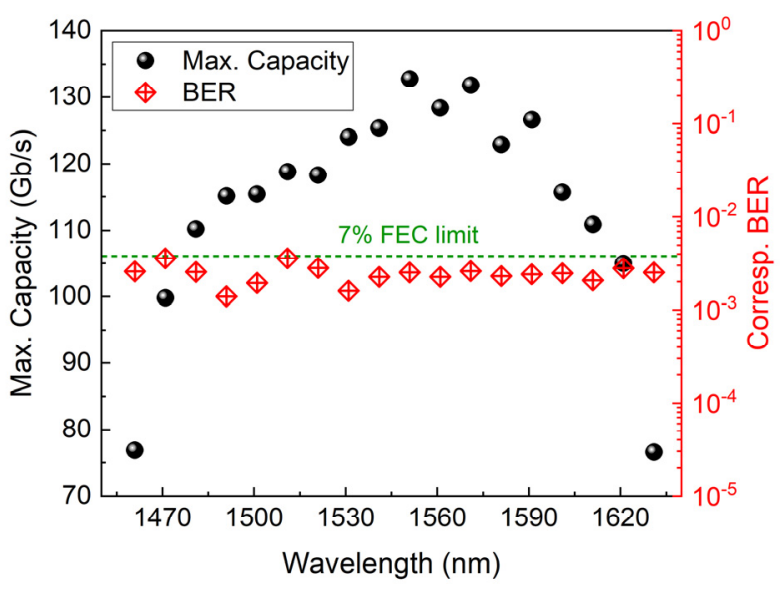

Fig. 12. Maximum capacity and the corresponding BER versus wavelength.
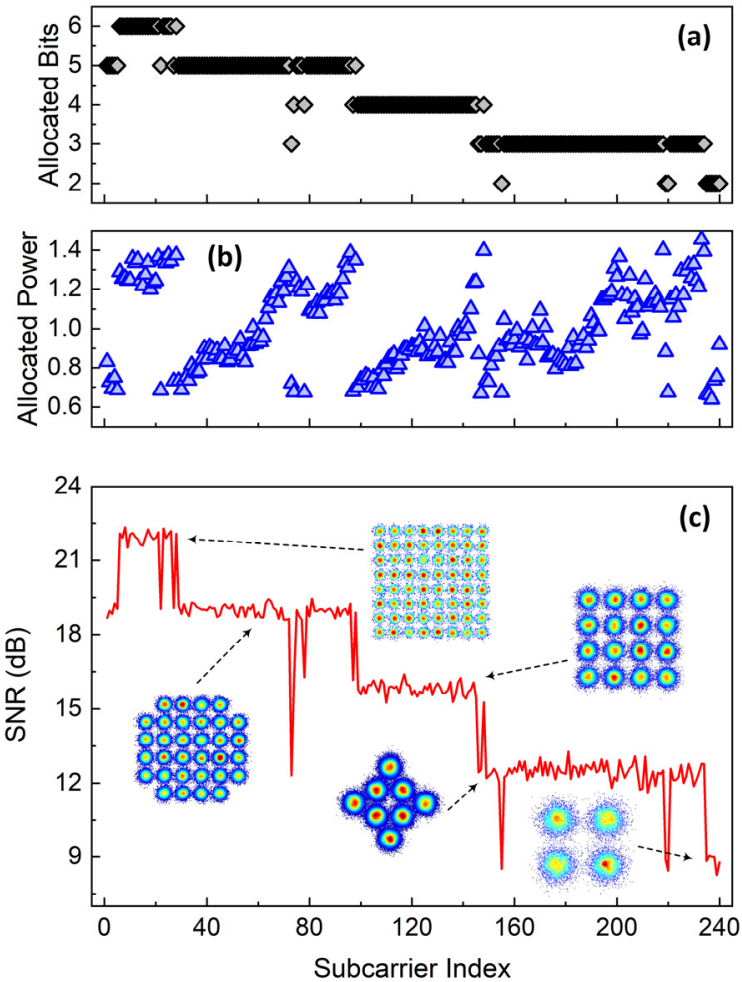

Fig. 13. (a) Bit allocation, (b) power allocation, and (c) SNR profile of the adaptively-loaded DMT transmission at $1551 \mathrm{~nm}$.

For reference, the bit and power allocation results and the corresponding SNR performance of the adaptively-loaded DMT transmission at the maximum capacity channel $(1551 \mathrm{~nm})$ are presented in Fig. 13. Generally, more bits were allocated to low-frequency subcarriers where higher SNRs were achievable. To maintain similar BERs across subcarriers that were loaded with the same number of bits, the power allocation profile exhibited a saw-tooth behavior, as expected. Consequently, the SNR profile of the adaptively-loaded DMT transmission was staircase-like, as shown in Fig. 13(c). The corresponding constellation diagrams that associate to different SNR levels are also given in Fig. 13(c). 


\section{Comparison with the $B 2 B$}

As indicated in prior works (e.g., [21, 32]), additional penalties on ROP sensitivity and/or BER/SNR performance may arise relative to the $\mathrm{B} 2 \mathrm{~B}$ when mode coupling to higher modes occurs in HCFs, i.e. when the fiber is not single-mode. Therefore, to validate the modal purity of the NANF, comparisons were conducted between the NANF transmission and B2B transmission at $1551 \mathrm{~nm}$, in which both the DD-OFDM ( $100 \mathrm{~Gb} / \mathrm{s})$ and the adaptively-loaded DMT were considered. For the B2B transmission, an optical attenuator with an equivalent loss was employed. An EDFA was used at the input of the NANF/attenuator to accommodate the variation of the launch power. Fig. 14 shows the comparisons in BER and average SNR of the 8QAM-DD-OFDM transmission, which corresponded to a data rate of $98.4375 \mathrm{~Gb} / \mathrm{s}$. It is clear that nearly identical BER and SNR performance compared to the B2B was achieved after transmission in the 1-km length of NANF, indicating that the penalty on transmission was negligible. Furthermore, in both the B2B and NANF cases, performance degradation was observed when the launch power was higher than $6 \mathrm{dBm}$. This was caused by the limited input power range of the high-gain $\mathrm{PD}$, which is integrated with a transimpedance amplifier.

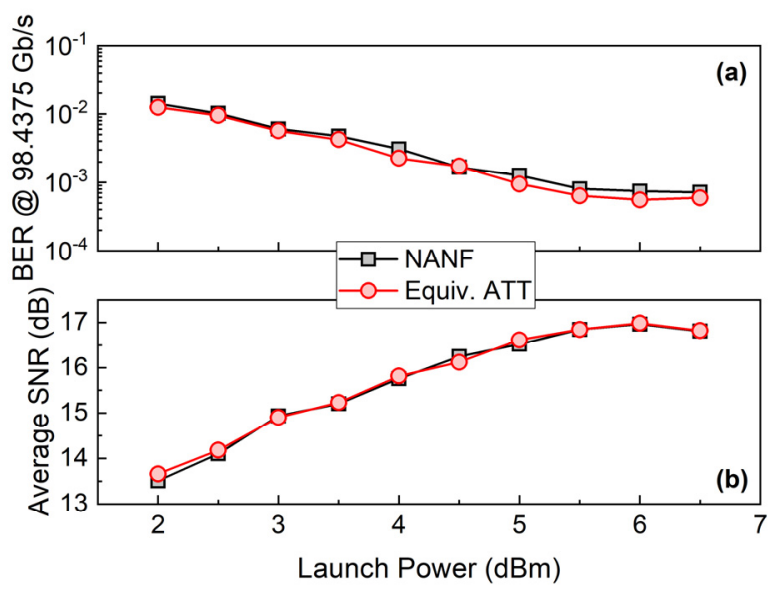

Fig. 14. (a) BER comparison with B2B at $1551 \mathrm{~nm}$, and (b) the corresponding average SNR versus launch power.

We also compared the maximum achievable capacity between the NANF transmission and B2B, where the adaptive bit-and-power loading was used to maximize the system's data rate at the 7\% FEC limit. The corresponding results are shown in Fig. 15. It is seen that similar capacities compared to the B2B could also be achieved after transmission in the NANF across the launch power range considered. Meanwhile, the BERs of both cases were also comparable and were all lower than the $7 \%$ FEC limit.

The results of both the $\sim 100-\mathrm{Gb} / \mathrm{s}$ DD-OFDM transmission and the adaptively-loaded DMT transmission confirm the excellent modal purity of the NANF. Moreover, the results of the adaptively-loaded DMT transmission indicate that the data rates demonstrated in our experiments were intrinsically restricted by the transceiver's electrical bandwidth rather than the fiber's performance.

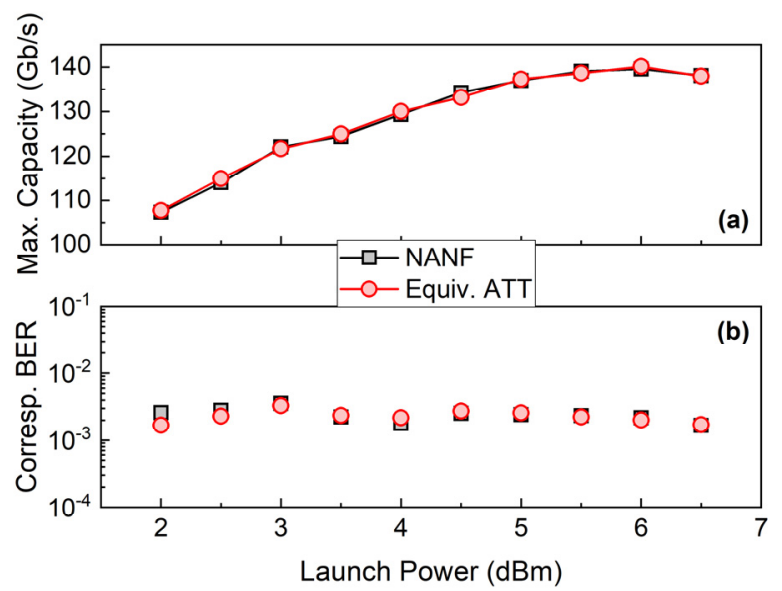

Fig. 15. (a) Comparison of maximum capacity with B2B at $1551 \mathrm{~nm}$, and (b) the corresponding BER versus launch power.

\section{CONCLUSION}

In this paper, we have demonstrated beyond $100-\mathrm{Gb} / \mathrm{s} / \lambda$ DD transmission over a $\sim 1-\mathrm{km}$ length of ultrawide bandwidth NANF. Two different transmission experiments were carried out to validate the ultrawide bandwidth and excellent modal purity of the fabricated fiber. In the first experiment, eight-channel dual-band $(\mathrm{O}+\mathrm{C})$ 100-Gb/s PAM4 WDM transmission was achieved using an in-house built BDFA and a commercial EDFA for optical pre-amplification at the receiver for the $\mathrm{O}$ - and $\mathrm{C}$-band respectively. To the best of our knowledge, this demonstration constitutes the highest reported transmission capacity in a DD HCF-based system. In the second experiment, we demonstrated beyond $100-\mathrm{Gb} / \mathrm{s} / \lambda$ adaptively-loaded DMT transmission over the $\mathrm{S}+\mathrm{C}+\mathrm{L}-$ bands in the NANF without using optical amplification. We capitalized on the excellent modal purity of the NANF, which allowed $>100-\mathrm{Gb} / \mathrm{s}$ penalty-free transmission over an ultrawide bandwidth. Very recent results have shown that more than one order of magnitude loss reduction in NANF as well as a significantly longer span length are achievable with further improvements subject to further realistic refinements in fabrication [23, 27]. The demonstrated results in this work are indicative of the promising potential provided by the combination of novel fiber and amplification technologies, which offer the prospect of reshaping high-speed optical interconnects in the years to come.

\section{ACKNOWLEDGMENT}

The data for this work is accessible through the University of Southampton Institutional Research Repository (DOI: https://doi.org/10.5258/SOTON/D1145).

\section{REFERENCES}

[1] E. Agrell, M. Karlsson, A.R. Chraplyvy, et al., "Roadmap of Optical Communications," J. Opt., vol. 18, pp. 1-40, 2016. 
[2] J. Cheng, C. Xie, Y. Chen, et al., "Comparison of Coherent and IMDD Transceivers for Intra Datacenter Optical Interconnects," in Proc. of OFC, San Diego, USA, paper W1F.2, 2019.

[3] R. Urata, H. Liu, X. Zhou, et al., "Data center Interconnect and Networking: from Evolution to Holistic Revolution," in Proc. of OFC, Los Angeles, USA, paper W3G.1, 2017

[4] K. Zhong, X. Zhou, J. Huo, et al., "Digital Signal Processing for Short-Reach Optical Communications: A Review of Current Technologies and Future Trends," J. Light. Technol., vol. 36, no. 2, pp. 377-400, 2018

[5] X. Pang, O. Ozolins, L. Zhang, et al., "Beyond 200 Gbps per Lane Intensity Modulation Direct Detection (IM/DD) Transmissions for Optical Interconnects: Challenges and Recent Developments," in Proc. of OFC, San Diego, USA, paper W4I.7, 2019.

[6] K. Kikuchi, "Fundamentals of Coherent Optical Fiber Communications," J. Light. Technol., vol. 34, no. 1, pp. 157-179, 2016.

[7] M. Chagnon, D.V. Plant, "504 and 462 Gb/s Direct Detect Transceiver for Single Carrier Short-reach Data Center Applications," in Proc. of OFC, Los Angeles, United States, paper W3B.2, 2017.

[8] L. Zhang, X. Hong, X. Pang, et al., "Nonlinearity-aware $200 \mathrm{Gbit} / \mathrm{s}$ DMT Transmission for C-band Short-reach Optical Interconnects with A Single Packaged Electro-absorption Modulated Laser," Opt. Lett., vol. 43, no. 2 , pp. 182-185, 2018.

[9] Y. Kai, M. Nishihara, T. Tanaka, et al., "Experimental Comparison of Pulse Amplitude Modulation (PAM) and Discrete Multi-tone (DMT) for Short-Reach 400-Gbps Data Communication," in Proc. of ECOC London, UK, paper Th.1.F.3, 2013.

[10] S. Kanazawa, H. Yamazaki, Y. Nakanishi, et al., "Transmission of 214-Gbit/s 4-PAM Signal using An Ultra-broadband Lumped-electrode EADFB Laser Module," in Proc. of OFC, Anaheim, USA, paper Th5B.3 2016.

[11] S. Lange, S. Wolf, J. Lutz, et al., "100 GBd Intensity Modulation and Direct Detection with an InP-Based Monolithic DFB Laser Mach-Zehnder Modulator," J. Light. Technol., vol. 36, no. 1, pp. 97-102, 2018

[12] P.D. Heyn, V.I. Kopp, S.A. Srinivasan, et al., "Ultra-dense $16 \times 56 \mathrm{~Gb} / \mathrm{s}$ NRZ GeSi EAM-PD Arrays Coupled to Multicore Fiber for Short-reach 896Gb/s Optical links," in Proc. of OFC, Los Angeles, USA, paper Th1B.7, 2017

[13] D.L. Butler, "Space-Division Multiplexing (SDM) Technology for Short Reach Fiber Optic Systems," in Proc. of OFC, Anaheim, USA, paper Tu3I.1, 2016.

[14] T. Hayashi, T. Nakanishi, K. Hirashima, et al., " $125-\mu m-C l a d d i n g$ Eight-Core Multi-Core Fiber Realizing Ultra-High-Density Cable Suitable for O-Band Short-Reach Optical Interconnects," J. Light. Technol., vol. 34, no. 1, pp. 85-92, 2016.

[15] S. Beppu, H. Takahashi, T. Gonda, et al., "56-Gbaud PAM4 Transmission over 2-km 125- $\mu \mathrm{m}$-Cladding 4-Core Multicore Fibre for Data Centre Communications," in Proc. of ECOC, Gothenburg, Sweden, paper Th.2.A.2, 2017

[16] F. Poletti, N. V. Wheeler, M. N. Petrovich, et al., "Towards High-capacity Fibre-optic Communications at the Speed of Light in Vacuum," Nat. Photon., vol. 7, pp. 279-284, 2013.

[17] D.J. Richardson, N.V. Wheeler, Y. Chen, et al., "Hollow Core Fibres and their Applications," in Proc. of OFC, Los Angeles, USA, paper Tu3H.1, 2017.
[18] F. Poletti, "Nested Antiresonant Nodeless Hollow Core Fiber," Opt. Express, vol. 22, no. 20, pp. 23807-23828, 2014.

[19] V.A.J.M. Sleiffer, Y. Jung, N.K. Baddela, et al., "High Capacity Mode-Division Multiplexed Optical Transmission in a Novel 37-cell Hollow-Core Photonic Bandgap Fiber," J. Light. Technol., vol. 32, no. 4, pp. 854-863, 2014

[20] Z. Liu, B. Karanov, L. Galdino, et al., "Nonlinearity-Free Coherent Transmission in Hollow-Core Antiresonant Fiber," J. Light. Technol., vol. 37, no.3, pp. 909-916, 2019.

[21] B.J. Mangan, M. Kuschnerov, J.W. Nicholson, et al., "First Demonstration of Hollow-core Fiber for Intra Data Center Low Latency Connectivity with A Commercial 100Gb/s Interface," in Proc. of OFC, Los Angeles, USA, paper M3D.4, 2015.

[22] M. Kuschnerov, V.A.J.M. Sleiffer, Y. Chen, et al., "Data Transmission through up to $74.8 \mathrm{~km}$ of Hollow-Core Fiber with Coherent and Direct-Detect Transceivers," in Proc. of ECOC, Valencia, Spain, paper Th. 1.2.4, 2015.

[23] A. Nespola, S. Straullu, T. D. Bradley, et al., "Record PM-16QAM and PM-QPSK Transmission Distance (125 and $340 \mathrm{~km})$ over Hollow-Core-Fiber," in Proc. of ECOC, Dublin, Ireland, paper PD1.5, 2019.

[24] H. Sakr, T.D. Bradley, Y. Hong, et al, "Ultrawide Bandwidth Hollow Core Fiber for Interband Short Reach Data Transmission," in Proc. of $O F C$, San Diego, USA, paper Th4A.1, 2019.

[25] Y. Hong, N. Taengnoi, K. R. H. Bottrill, et al., "Dual O+C-band WDM Transmission over 1-km Hollow Core NANF using An O-band Bismuth-doped Fibre Amplifier," in Proc. of ECOC, Dublin, Ireland, paper Tu.2.E.1, 2019.

[26] Y. Hong, K. R. H. Bottrill, N. Taengnoi, et al.,“ Beyond 100-Gb/s/ $\lambda$ Direct-detection Transmission over the S+C+L-bands in An Ultra-wide Bandwidth Hollow Core Fibre," in Proc. of ECOC, Dublin, Ireland, paper Th.2.E.5, 2019.

[27] T. D. Bradley, G. T. Jasion, J. R. Hayes, et al., "Antiresonant Hollow Core Fibre with $0.65 \mathrm{~dB} / \mathrm{km}$ Attenuation across the $\mathrm{C}$ and $\mathrm{L}$ Telecommunication Bands," in Proc. of ECOC, Dublin, Ireland, paper PD3.1, 2019.

[28] H. Sakr, Y. Hong, T.D. Bradley, et al, "Interband Short Reach Data Transmission in Ultrawide Bandwidth Hollow Core Fiber," J. Light. Technol., early access, 2019.

[29] N. K. Thipparapu, A. A. Umnikov, P. Barua, et al., "Bi-doped Fiber Amplifier with A Flat Gain of $25 \mathrm{~dB}$ Operating in the Wavelength Band 1320-1360 nm," Opt. Lett., vol. 41, no. 7, pp. 1518-1521, 2016.

[30] Y. Hong, T. D. Bradley, N. Taengnoi, et al., "Comparative Investigations between SSMF and Hollow-core NANF for Transmission in the S+C+L-bands," in Proc. of OFC, San Diego, USA, paper W3E.3, 2020.

[31] P. S. Chow, J. M. Cioffi, and J. A. C. Bingham, "A Practical Discrete Multitone Transceiver Loading Algorithm for Data Transmission over Spectrally Shaped Channels," IEEE Trans. on Commun., vol. 73, no. 2 , pp. 773-775, 1995.

[32] H. Zhang, N. Kavanagh, Z. Li, et al., "100 Gbit/s WDM Transmission at 2 $\mu \mathrm{m}$ : Transmission Studies in Both Low-loss Hollow Core Photonic Bandgap Fiber and Solid Core Fiber," Opt. Express, vol. 23, no. 4, pp. 4946-4951, 2015 